\title{
On Optimality Conditions in some Control Problems for Memory Kernels in Viscoelasticity
}

\author{
L. VON WOLFERSDORF
}

'The inverse problem of identifying the memory kernel in the stress-strain-relation of Boltzmann type for a rod is reduced to an optimal control problem with a quadratic cost functional in the state for the related integro-differential wave equation. For this problem necessary optimality conditions are derived and transformed into explicit form by means of suitably defined adjoint state functions.

Key words: Identification, optimal control, memory kernels AMS subject classification: $45 \mathrm{~K} 05,49 \mathrm{~B} 22,73 \mathrm{~F} 05$

Recently papers by M. Grasselli, S.I. Kabanikhin, A. Lorenzi and others appeared in the literature dealing with the identification of memory kernels in the linear theory of viscoelasticity by solving a corresponding inverse problem, see for instance [1 - 3]. Following the usual approach in estimating unknown parameters with the help of a related minimum condition $Y$. Yanno [6] and the author [5] reduced some identification problems of this kind to optimal control problems for the corresponding wave equation. Whereas Yanno deals with the creep memory kernel in an infinite interval with additional informa. tion about the strain, we consider the relaxation memory kernel in a finite interval with additional information about the displacement itself.

In the present paper for the solutions of the control problems with the integrodifferential equation in [5] optimality conditions are derived by defining suitable adjoint state functions. 'Thereby the class of problems in [5] is slightly broadened.

\section{Formulation of problems}

As in [5] we deal with the following inverse problem for determining the displacement $u=u(x, t)$ and the memory kernel $m=m(t)$ in a viscoelastic rod:

$$
L u \equiv \rho \frac{\partial^{2} u}{\partial t^{2}}(x, t)-c^{2} \frac{\partial^{2} u}{\partial x^{2}}(x, t)+\int_{0}^{t} m(t-\tau) \frac{\partial^{2} u}{\partial x^{2}}(x, \tau) d \tau=\mathcal{X}(x, t)
$$

L. von Wolforedorf: Borgakad. Froiborg. FB Math., B.-von-Cotta-Str.2, D - 09596 Frolbers 
for $0<x<1.0<t<T$,

$$
\begin{array}{ll}
u(x, 0)=\varphi(x), \quad u_{t}(x, 0)=\psi(x) & \text { for } 0<x<1 \\
u(0, t)=u(1, t)=0 & \text { for } 0<t<T
\end{array}
$$

and additionally

$$
u\left(x_{0}, t\right)=h(t) \quad \text { for } \quad 0<t<T \text {, }
$$

where $x_{0} \in(0,1)$, fixed. Here $\rho$ is the mass density, $c^{2}$ the instantaneous stress modulus and $\mathcal{X}$ the outer force density. The wave equation (1) follows from the well-known Boltzmann stress-strain relation between the strain $\epsilon$ and the stress $\sigma$, the relation $\epsilon=\partial u / \partial x$ and the momentum equation $\rho\left(\partial^{2} u / \partial t^{2}\right)=\partial \sigma / \partial x+\chi$, where we restrict ourselves to the most important case of constant $\rho$ and $c^{2}$ for $m=m(t)$ (cp. [5]).

Instead of the point condition (4) we also prescribe more realistic additional conditions in integral form

$$
\int_{0}^{1} \gamma_{j}(x) u(x, t) d x=h_{j}(t) \quad(j=1, \ldots, q),
$$

where $\gamma_{j}$ are non-negative weight functions such that the integrals in (5) represent average displacements in the neighbourhood of $q$ points $x_{j} \in(0,1)$.

In the corresponding optimal control problems the additional conditions (4) and (5) are replaced by the minimum condition

$$
\min _{m \in M} H[m]
$$

where $M$ is a prescribed class of physically admissible kernels $m$ and

$$
H[m]=\|z-h\|^{2}, \quad z=u\left(x_{o}, \cdot\right),
$$

or

$$
H[m]=\sum_{j=1}^{q} \alpha_{j}\left\|\dot{z}_{j}-h_{j}\right\|^{2} . \quad\left(\alpha_{j}>0\right)
$$

with

$$
z_{j}=\int_{0}^{1} \gamma_{j}(x) u(x, \cdot) \cdot d x \quad\left(\gamma_{j} \in L_{2}(0,1)\right),
$$

respectively. Here $u=u[m]$ is the solution of (1) - (3) corresponding to $m$. The given functions $h, h_{j}$ are from $L_{2}(0, T)$ and $\|\cdot\|$ denotes the norm in $L_{2}(0, T)$.

At first we recall some results from [5] on the solvability of the initial-value problem (1) - (3) which are based on the method in [4: Chap. 6, \$2] and on the optimal control problem (6), (7).

For given absolutely continuous kernel $m$ in $[0, T]$ and data $\varphi \in H_{\circ}^{1}(0,1), \dot{\psi} \in L_{2}(0,1)$, $\mathcal{X} \in L_{2}(Q), Q=(0,1) \times(0, T)$ the problem (1) - (3) has a unique generalized solution $u \in H^{\prime}(Q)$ which can be represented by a Fourier series

$$
u(x, t)=\sum_{n=1}^{\infty} A_{n}(t) \sin n \pi x
$$


converging in $H^{1}(Q)$. If $M$ is a compact subset of the space $A C$ of absolutely continuous functions on $[0, T]$, then the optimal control problem $(6),(7)$ has an optimal solution $m_{0}$. Further the functional (7) possesses the Fréchet derivative $H^{\prime}[m]$ defined by

$$
H^{\prime}[m](k)=2 \int_{0}^{T} F(t) U_{1}\left(x_{0}, t\right) d t, \quad F(t) \doteq z(t)-h(t),
$$

where $U_{1} \in H^{1}(Q)$ is the generalized solution of the equation

$$
L U_{1}(x, t)=-\int_{0}^{t} k(t-\tau) u_{x x}(x, \tau) d \tau
$$

with homogeneous initial and boundary conditions.

The existence of an optimal control for the problem (6), (7) follows from the proof in [5] of the (indeed Lipschitz) continuity of the operator $A: M \rightarrow L_{2}(0, T)$ defined by $A m=u\left(x_{o}, \cdot\right)$, where $u$ is the generalized solution of (1) - (3) corresponding to the kernel $m$ in (1). Using Schwarz's inequality this proof extends to the operators $A_{j}: M \rightarrow L_{2}(0, T)$ defined by $A_{j} m=\int_{0}^{1} \gamma_{j}(x) u(x, \cdot) d x$. Therefore the existence of an optimal control also holds true for the problem (6), (8).

In the same way the proof in [5] of the Fréchet differentiability of the operator $A$ carries over to the operators $A_{j}$ such that the functional (8) has the Fréchet derivative $H^{\prime}[m]$ given by

$$
H^{\prime}[m](k)=2 \int_{Q} F(x, t) U_{1}(x, t) d x d t
$$

with

$$
F(x, t)=\sum_{j=1}^{q} \alpha_{j} \gamma_{j}(x)\left[z_{j}(t)-h_{j}(t)\right] \in L_{2}(Q) .
$$

\section{Optimality conditions}

Let now $M$ be a convex subset of the space $A C$. Then there holds the optimality condition

$$
H^{\prime}\left[m_{o}\right]\left(m-m_{o}\right) \geq 0 \quad \text { for all } m \in M
$$

for an optimal solution $m_{0}$ of problem $(6),(7)$ and $(6),(8)$, respectively. This means

$$
\int_{0}^{T} F_{o}(t) U_{1}\left(x_{o}, t\right) d t \geq 0 \quad \text { for all } m \in M
$$

and

$$
\int_{Q} F_{0}(x, t) U_{1}(x, t) d x d t \geq 0 \quad \text { for all } m \in M
$$


respectively. where $\digamma_{0}=u_{0}\left(x_{0} \cdot\right)-h \in L_{2}(0 . T)$ and

$$
F_{0}(s, t)=\sum_{j=1}^{\varphi} n_{j} \gamma_{j}(x)\left[z_{j}^{o}(t)-h_{j}(t)\right] \in L_{2}(Q)
$$

with $z_{j}^{o}(t)=\int_{0}^{1} \gamma_{j}(x) u_{o}(x . t) d x$ and $u_{i} \in H^{1}(Q)$ is the solution of (1) - (3) corresponding to $m_{n}$. Analogously, $U_{1}$ is related to this solution $u_{0}$ of (1) - (3) and the increment $k=m-m_{0}$ in $(12)$.

We transform the conditions (16) and (17) by means of an adjoint state function $\psi$ defined as generalized solution from $H^{1}(Q)$ of the adjoint equation to (1)

$$
I^{*} \psi^{\prime} \equiv \rho \frac{\partial^{2} \psi^{\prime}}{\partial t^{2}}(x . t)-c^{2} \frac{\partial^{2} \psi}{\partial x^{2}}(x . t)+\int_{l}^{T} m_{o}(\tau-t) \frac{\partial^{2} \psi}{\partial x^{2}}(x, \tau) d \tau=\left\{\begin{array}{l}
F_{o}(t) \delta\left(x-x_{o}\right) \\
F_{o}(x, t)
\end{array}\right.
$$

in (Q. respertively, with homogenous boundary and terminal conditions. I.e., $\psi \in H^{1}(Q)$ fulfils the conditions $\psi(0, t)=\psi(1, t)=0$ and $\psi(x, T)=0$ and the integral identity

$$
\begin{array}{r}
\int_{Q}\left[c^{2} V_{x} J_{x}-\int_{t}^{T} m_{0}(\tau-t) \psi_{x}(x, \tau) d \tau \cdot U_{x}-\rho U_{t} \psi_{t}\right] d x d t \\
= \begin{cases}\int_{0}^{T} F_{0}(t) U\left(x_{0}, t\right) d t & \text { in case of }(6),(7) \\
\int_{Q} F_{0}(x, t) U(x, t) d x d t & \text { in case of }(6),(8)\end{cases}
\end{array}
$$

for any $U \in H^{\prime}(Q)$ with $U(0, t)=U(1, t)=0$ and $U(x, 0)=0$. Since $F_{o} \in L_{2}(Q)$ in case of problem (6), (8) the existence of $\psi \in H^{1}(Q)$ to (19) follows from the considerations in [.5] as for the state function $u$. In case of problem (6), (7) we have

$$
\forall(r, t)=\sum_{n=1}^{\infty} i_{n}(t) \sin n \pi x
$$

where

$$
\rho \bar{C}_{n}(l)+c^{2} \pi^{2} n^{2} C_{n}(l)=\pi^{2} n^{2} \int_{l}^{T} C_{n}(\tau) n_{u}(\tau-t) d \tau+F_{n}(t)
$$

with $F_{n}=2 \sin n \pi x_{0} \cdots F_{0} \in L_{2}(0, T)$ and $C_{n}^{\prime}(T)=\dot{C}_{n}(\dot{T})=0$. The functions $C_{n}$ satisfy the Volterra integral equations

$$
C_{n}^{\prime}(t)-\lambda_{n}\left(N_{n} C_{n}^{\prime}\right)(t)=\Psi_{n}(t),
$$

where $\lambda_{n}=c \pi n / \sqrt{\rho}$,

$$
\Psi_{n}(t)=\frac{1}{\rho} \frac{1}{\lambda_{n}} \int_{1}^{T} F_{n}(s) \sin \left[\lambda_{n}(s-t)\right] d s
$$

and

$$
\left(N_{n} C_{n}\right)(\ell)=\int_{l}^{T} C_{n}(\tau) p_{n}(l, \tau) d \tau
$$


with the kernels

$$
p_{n}(t, \tau)=\frac{1}{c^{2}} \int_{l}^{\tau} m_{0}(\tau-s) \sin \left[\lambda_{n}(s-t)\right] d s .
$$

Since $m_{0} \in A C$ the kernels $\lambda_{n} p_{n}$ are uniformly bounded and there holds the estimation (cp. [5])

$$
\max _{t \in[0, T]}\left|C_{n}^{\prime}(t)\right| \leqq \text { Const } \max _{t \in[0, T]}\left|\Psi_{n}(t)\right|
$$

and hence from (23) there follows $C_{n}=O(1 / n)$ and after an integration by parts in (23) $C_{n}=O\left(1 / n^{2}\right)$ if additionally $F_{o} \in A C$ is fulfilled. Further

$$
\max _{t \in[0, t]}\left|\dot{C}_{n}(t)\right| \leqq \max _{t \in[0, T]}\left|\dot{\Psi}_{n}(t)\right|+\text { Const } \lambda_{n} \max _{t \in[0, T]}\left|C_{n}(t)\right|
$$

such that $\dot{C}_{n}=O(1 / n)$ for $F_{o}^{\prime} \in A C$, too. Therefore we have $\psi \in L_{2}(Q)$ in general and $\psi \in H^{1}(Q)$ under the additional assumptions $h \in A C^{\prime}$ and $u_{o}\left(x_{o}, \cdot\right) \in A C$.

The function $U_{1} \in H^{1}(Q)$ fulfils the integral identity

$$
\begin{array}{r}
\int_{Q}\left[c^{2} U_{1 x} v_{x}-\int_{0}^{t} m_{o}(t-\tau) U_{1 x x}(x, \tau) d \tau v_{x}-\rho U_{1 t} v_{t}\right] d x d t=\int_{Q} r v_{x} d x d t \\
r(t)=\int_{0}^{t} k(t-\tau) u_{o x}(x, \tau) d \tau
\end{array}
$$

for any $v \in H^{1}(Q)$ with $v(0, t)=v(1, t)=0$ and $v(x, 7)=0$. Taking the difference of (19) for $U=U_{1}$ and (25) for $v=\psi$, we obtain the optimality conditions (16), (17) in the desired form

$$
\int_{0}^{T} H(s)\left[m-m_{0}\right](s) d s \geq 0 \quad \text { for all } m \in M,
$$

where

$$
H(s)=\int_{0}^{T} \int_{0}^{1} \psi_{x}(x, l) u_{o x}(x, t-s) d x d t .
$$

Theorem: For the problems (6), (7) and (6), (8) the optimality condition (26) with (27) holds, where the adjoint state function $\psi \in H^{1}(Q)$ is defined by the identity (19) and in case of problem (6), (7) the additional conditions $h \in A C$ and $u_{0}\left(x_{0}, \cdot\right) \in A C$ are assumed.

Remark: Using the Fourier expansion (20) for $\psi$ and the corresponding one for $U_{1}$ it can be easily shown that the integrals in (16) and (26) are equal, where

$$
H(s)=\frac{\pi^{2}}{2} \sum_{n=1}^{\infty} n^{2} \int_{0}^{T} A_{n}(t-s) C_{n}(t) d t
$$


with $A_{n}$ the Fourier coefficients of $u_{o}$ in (10). Therefore if $u_{o x x} \in L_{2}(Q)$, the optimality condition for problem (6), (7) can be taken also in the form (26) with

$$
H(s)=-\int_{0}^{T} \int_{0}^{1} \psi(x, t) u_{o x x}(x, t-s) d x d t
$$

where the adjoint state function $\psi \in L_{2}(Q)$ is now simply defined by (20) with (21) or (22) or equivalently as a function $\psi \in L_{2}(Q)$ satisfying the identity

$$
\int_{Q} \psi L U(x, t) d x d t=\int_{0}^{T} F_{\mathrm{o}}(t) U\left(x_{o}, t\right) d t
$$

for any $U \in H^{2}(Q)$ with $U(0, t)=U(1, t)=0$ and $U(x, 0)=0$. From (16) and (30) for $U=U_{1}$ with (12) the condition (26) with (29) also follows in a direct way. The additional assumption $u_{o x x} \in L_{2}(Q)$ or $u_{o} \in H^{2}(Q)$ is fulfilled if we have data $\varphi \in H^{2}(0,1)$, $\psi \in H^{1}(0,1), \mathcal{X} \in H^{1}(Q)$ with $\varphi=\psi=\mathcal{X}=0$ for $x=0,1$ in problem (1) - (3), for instance (cp. [5]). (A corresponding remark is also true for problem (6), (8), of course.)

\section{REFERENCES}

[1] Grasselli, M.: On an inverse problem for a linear hyperbolic integrodifferential equation. Forum Math. 1 (1993) (to appear).

[2] Grasselli, M., Kabanikhin, S.I. and A. Lorenzi: An inverse hyperbolic integrodifferential problem arising in Geophysics I (in Russian). Sibirsk. Math. Zh. 33 (1992), 58 68.

[3] Grasselli, M., Kabanikgin, S.I. and A. Lorenzl: An inverse hyperbolic integrodifferential problem arising in Geophysics II. Nonlin. Anal., Theory, Meth. Appl. 15 (1990), 283 $-298$.

[4] Miknailov, V.P.: Partial Differential Equations (in Russian). Moscow: Nauka 1976.

[5] V. Wolfersdorf, L.: On identification of memory kernels in linear viscoelasticity. Math. Nachr. 161 (1993), $203-217$.

[6] Yanno, Y.: On an inverse problem for a hyperbolic equation (in Russian). Uch. Zap. Tartu Gos. Univ. 672 (1984), 40 - 46.

Received 22.03.1993 